\title{
Innovación y cambio microinstitucional en el sector salud: evidencia de la telemedicina en México*
}

\author{
José Luis Sampedro Hernández**
}

\section{RESUMEN}

Este artículo contribuye a comprender cómo influye la adopción y absorción de nuevas tecnologías, en especial la telemedicina, en el cambio de las estructuras técnicas y cognitivas de las organizaciones de salud y en las percepciones y toma de decisiones de sus especialistas. Esto afecta la configuración de los procesos de aprendizaje, innovación y cambio microinstitucional del sector público de la salud, particularmente en unidades de primer nivel. Se analiza cómo estos procesos influyen en los servicios de atención médica. Se busca explicar cómo influye la adopción y absorción de nuevas tecnologías en la reconfiguración de los procesos de aprendizaje colaborativo, la creación y transformación del conocimiento y los modos de coordinación de los distintos cúmulos de información y conocimiento, lo que afecta a los servicios de salud. Se argumenta que la telemedicina es una tecnología social que permite la difusión de información y conocimiento específico para la toma de decisiones críticas y complejas en un menor tiempo, afectando el desarrollo de habilidades, conocimiento y modos de coordinación. Se realiza un estudio de caso múltiple-exploratorio. Los casos a estudiar son unidades médicas de la Secretaría de Salud en Oaxaca, Chiapas y Guerrero; la unidad de análisis se refiere a los proyectos de telemedicina implementados. El trabajo de campo se realizó entre junio de 2011 y enero de 2012.

Palabras clave: conocimiento, innovación, aprendizaje colaborativo, modos de coordinación, cambio microinstitucional, telemedicina.

Clasificación JEL: 033, I1.

\begin{abstract}
This paper contributes to understand how the adoption and absorption of new technologies, in particular telemedicine, influence on change of technical and cognitive structures of healthcare units, and on changes of perceptions and making decisions. This change affect the configuration of learning, innovations and micro-institutional processes. It also analyzes how these processes influence on healthcare services. We will try to explain how the adoption of new technologies influence on the reconfiguration of learning processes, on the creation of new knowledge, and on the modes of coordination of different cumuli of information and knowledge, and how that reconfiguration influences on the healthcare services. We suggest that telemedicine is a social technology that allows transferring of specific knowledge and information in order to take critical and complex decisions in a short time, affecting the development of skills, knowledge, and the modes of coordination among the actors. It will be illustrated by an exploratory multiple-case study. The cases are medical units that internalized telemedicine services in Oaxaca, Chiapas and Guerrero. In these cases, telemedicine projects are the unit of analysis. The fieldwork was done between June of 2011 and January of 2012.
\end{abstract}

Keywords: knowledge, innovation, collaborative learning, coordination modes, microinstitutional change, telemedicine.

JEL classification: 033 , I1.

* Fecha de recepción: 11/07/2012. Fecha de aprobación: 06/06/2013.

** Profesor-investigador del Departamento de Estudios Institucionales de la Universidad Autónoma Metropolitana-Cuajimalpa.Correo electrónico: sampedroh@yahoo.com.mx.

Este documento es parte del proyecto de investigación "Revaloración de la dimensión meso en los 


\section{INTRODUCCIÓN}

El sistema público de salud mexicano enfrenta problemas estructurales como la falta de recursos financieros y tecnológicos, una baja cobertura e ineficiencia en sus servicios, cambio demográfico y epidemiológico y, derivado de esto, alteraciones en la demanda de servicios de atención a la salud. La mayoría de los recursos hospitalarios están concentrados en las grandes ciudades y llevarlos al resto del país resulta costoso, tanto en el caso de infraestructura como de especialistas. Ante la complejidad de estos retos hay diversas estrategias para atenderlos parcialmente. Por ejemplo, la incorporación de nuevas tecnologías tiene efectos importantes en los procesos técnicos, en las percepciones y en la toma de decisiones de los médicos, así como en los procesos organizacionales de los hospitales.

En la actualidad existe un amplio espectro de tecnologías médicas que se incorporan al sector salud para mejorar e incrementar sus servicios. El expediente clínico electrónico y la telemedicina son nuevas tecnologías que se han implementado parcial y lentamente para apoyar los procesos de prevención, diagnóstico, tratamiento, monitoreo y seguimiento de diversas enfermedades. $\mathrm{Su}$ uso también influye en otros procesos relacionados, como la educación médica por medio de la socialización del conocimiento y la información, y en los procesos de gestión interna de las unidades médicas.

La absorción de estas tecnologías implica cambios al interior de las organizaciones que se reflejan en las estructuras técnicas y cognitivas de los actores al moldear y cambiar las percepciones (individuales, grupales y organizacionales) y en la toma de decisiones, ${ }^{1}$ además de cambios externos derivados de los primeros.

Esto implica una transformación de los procesos de aprendizaje, de la transferencia, difusión y explotación de diversos cúmulos de información y conocimiento, y al mismo tiempo una modificación en los modos de coordinación entre los actores, es decir, hay cambios en los procesos que afectan la capacidad de innovación y cambios microinstitucionales.

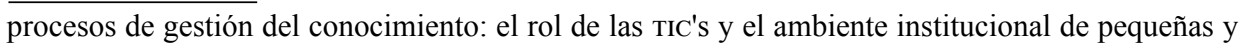
medianas empresas", desarrollado en la UAM-C y financiado por CONACYT (proyecto núm.133206).

1 “La percepción de los problemas desempeñan un papel relevante en cualquier etapa del cambio institucional [...] si bien hay actores clave (como los emprendedores institucionales) que son cruciales en la construcción social de tales percepciones, las dimensiones cognitivas de los contextos institucionales limitan las opciones de los actores" (Campbell, 2009). 
En este sentido, el objetivo del presente trabajo es explorar cómo la adopción y absorción de nuevas tecnologías influye en los cambios de las estructuras técnicas y cognitivas de las organizaciones de salud y en las percepciones y la toma de decisiones de los especialistas del área. Tales transformaciones tienen importantes implicaciones en la configuración de los procesos de aprendizaje, innovación y cambio microinstitucional de una parte importante de las unidades médicas del sector público de la salud, particularmente en aquellas de atención médica básica, llamadas de primer nivel. Al mismo tiempo, se analiza la manera en que estos procesos influyen en los servicios de atención médica en el sistema de salud mexicano.

Las preguntas centrales son: ¿cómo influyen la adopción y absorción de nuevas tecnologías en la reconfiguración de los procesos de aprendizaje, en la creación de nuevo conocimiento y en los patrones de coordinación de los diferentes cúmulos de información y conocimiento? ¿Cómo afecta esta reconfiguración a los servicios de salud?

La estrategia de investigación se basa en un estudio de caso múltipleexploratorio. Se realizaron 14 entrevistas semiestructuradas a líderes de proyecto, médicos y personal de apoyo entre junio de 2011 y enero de 2012. Los casos a estudiar son unidades médicas ubicadas en Oaxaca, Chiapas y Guerrero, y la unidad de análisis está referida a los proyectos de telemedicina implementados.

Uno de los argumentos consiste en que la telemedicina es una tecnología social ${ }^{2}$ que permite la transferencia y difusión de información y conocimiento específicos, así como tomar decisiones críticas y complejas en menor tiempo, lo que afecta el desarrollo de habilidades y conocimiento de los médicos y la calidad de los servicios otorgados a través de la teleconsulta. Esto también influye en los modos de coordinación entre los actores, es decir, en un cambio microinstitucional, el cual ocurre a distintos niveles y con diferentes determinantes y dinámicas. Al mismo tiempo, esto se deja sentir en la calidad de los servicios de salud. En el nivel microinstitucional las nuevas tecnologías tienen un efecto dinámico de corto plazo en el rediseño de las estructuras técnicas y cognitivas del ámbito organizacional, con efectos marginales de cambio en los modos de coordinación.

La estructura del trabajo es la siguiente. En el primer apartado se discute el marco teórico conceptual considerando dos perspectivas de análisis: la evolu-

2 "Una tecnología social se caracteriza por las pautas de interacción [y coordinación] humana y no por la ingeniería física” (Nelson y Sampat, 2001). 
tiva y la institucional. Después, en la segunda sección, se presenta la metodología. En la tercera parte se plantean los elementos de contexto del sector público de salud en México, con énfasis en el tipo de tecnología médica incorporada en él. En el cuarto apartado se discuten los hallazgos de la investigación. Finalmente, se presentan las reflexiones finales y conclusiones.

\section{INNOVACIÓN Y CAMBIO INSTITUCIONAL: DOS PERSPECTIVAS ANALÍTICAS CONVERGENTES}

\section{De la difusión a la capacidad de absorción en los procesos de innovación}

La literatura sobre innovación ha avanzado desde múltiples campos del conocimiento para tratar de analizar cómo la organización económica desarrolla procesos de innovación que afectan su desempeño. El modelo lineal basado en la invención (investigación básica), innovación (investigación aplicada, desarrollo, fabricación industrial) y difusión (comercialización) se ha complementado con modelos más complejos basados en redes de colaboración inter- e intraorganizacional. En todo caso, el proceso de difusión y adopción posee marcos analíticos propios y muy amplios para explicar los procesos de transferencia/difusión y los de adopción y absorción de conocimiento y tecnologías. Una buena parte de la evidencia empírica de estos estudios se basa en el estudio de la empresa como una de las organizaciones más importantes en la generación de cambio técnico e innovación, y la mayoría de las teorías respaldan y validan la innovación (en un sentido amplio) considerando a la empresa como unidad de análisis.

Sin embargo, la innovación surge a partir de otros actores desvinculados en algún sentido del mercado y organizados bajo una lógica distinta a la de de la maximización de beneficios y la competencia económica. Las organizaciones del sector público, en general, tienen una lógica de comportamiento distinta a las del mercado: no buscan la ganancia ni establecen estrategias de competencia como medio de supervivencia y, a pesar de ello, en algún sentido rediseñan sus modos de organización del trabajo, de redistribución y de optimización de los recursos. De tal suerte, los distintos actores del sector público se insertan en dinámicas de cambio constante marcadas por las tendencias internacionales para lograr un sector público eficaz, eficiente, con calidad, sustentable e incluyente.

Particularmente en el sector salud, el constante avance de medicamentos e instrumental médico demanda de sus especialistas una actualización dinámica que implica el desarrollo de procesos de aprendizaje y recreación del conoci- 
miento en múltiples niveles. Al mismo tiempo, las organizaciones médicas requieren capacidades organizacionales, tecnológicas e institucionales para adoptar y absorber las tecnologías y el conocimiento generado en el entorno. Esta preocupación ha sido poco explorada en la amplia literatura sobre economía de la innovación, sin embargo, una parte importante de las categorías que se utilizan para analizar la organización económica son pertinentes para explicar el comportamiento innovador de las organizaciones públicas. Para Nugroho (2011), lo que importa no es la motivación del beneficio sino cómo se adoptan y difunden las ideas para lograr metas sociales más amplias.

La adopción tecnológica es un concepto aplicado que desarrolló Everett M. Rogers en 1962 en su libro seminal Diffusion of Innovation (Rogers, 2003); dicho texto describe el modelo de difusión basado en conceptos como innovadores, adoptadores tempranos de las innovaciones, mayoría temprana, y adoptadores tardíos y rezagados marcados por una alta incertidumbre. A pesar de ser ampliamente criticado, este modelo aún es vigente por sus implicaciones prácticas y teóricas para un cúmulo importante de innovaciones.

Durante la década de los sesenta se iniciaron las investigaciones que darían origen a una de las mayores innovaciones tecnológicas de la siguiente década, el microprocesador, y a la industria de las tecnologías de la información (incluidas las de hardware, de software y de servicios relacionados), que se desplegarían por todo el mundo con una tasa de adopción creciente entre las organizaciones económicas, públicas y de la sociedad civil, entre otras. De forma paralela, se desarrolló Internet, que se ha propagado en la sociedad a una velocidad que pocas tecnologías han logrado.

Una de las dificultades reside en que las organizaciones no sólo enfrentan la alta difusión y aglomeración de nuevas tecnologías sino la urgencia de desarrollar capacidades para identificar las que responden a sus necesidades e internalizar, socializar y explotar los cúmulos de conocimiento e información asociados a ellas; en otras palabras, enfrentan un problema de capacidad de absorción.

Este concepto, acuñado por Cohen y Levinthal (1990), se refiere a la habilidad de la empresa para evaluar información y conocimiento útil del entorno y, a su vez, asimilarlos y expresarlos en nuevos productos. Las organizaciones pueden incrementar su capacidad de absorción mediante la acumulación de experiencias o conocimiento e información específicos, pero para lograrlo deberán tener capacidades internas que les permitan identificar, internalizar y explotar conocimientos inéditos y expresarlos en nuevos productos, procesos y servicios (Sampedro, 2011; Lund, 2004; Lane, Koka y Pathak, 2002; Van den Bosch, Vol- 
berda y De Boer, 1999; Cohen y Levinthal, 1990). Diversos autores identifican varios factores para incrementar la capacidad de absorción de las organizaciones:

- Conocimiento previo: se parte del supuesto de que las organizaciones cuentan con una capacidad previa que les permite incrementar su capacidad de absorción a lo largo del tiempo (Cohen y Levinthal, 1990).

- Aprendizaje interorganizacional: significa que las organizaciones deben contar con sistemas de procesamiento de información y conocimiento similares a los de las organizaciones que juegan el rol de maestras o transmisoras de tecnología/conocimiento (Lane, Koka y Pathak, 2002)

- Aprendizaje intraorganizacional: implica que la organización debe contar con un "sistema de capacidades" (capacitación, foros de discusión, etcétera) que le permita integrar conocimiento explícito, así como procesos de coordinación y socialización de conocimientos entre los distintos grupos de trabajo (departamentos o áreas). La socialización está relacionada con la cultura de la organización y con el sistema de normas y valores (Lane, Koka y Pathak, 2002; Van den Bosch, Volberda y De Boer, 1999).

- Movilización de recursos organizacionales (de manera particular en la micro- y pequeña empresa): implica movilizar recursos organizacionales $\mathrm{y}$, con ello, la polivalencia de actividades y habilidades, lo cual, al mismo tiempo, limita la especialización (Jones y Craven, 2001).

- Interfaces internas y externas: significa crear mecanismos que permitan una adecuada identificación e internalización de la información y conocimiento específicos y fragmentarios que posee cada agente; mediante las interfaces se reducen las asimetrías cognitivas entre los agentes y al mismo tiempo son mecanismos de aprendizaje (Sampedro, 2011).

La transferencia de conocimiento puede involucrar el uso de uno o más de estos factores y entre mejor desarrollados estén, la organización tendrá una mayor capacidad de internalizar el conocimiento al momento de adquirirlo. En otras palabras, la transferencia implica para el receptor contar con los medios para identificar, implementar y adaptar, así como socializar y explotar adecuadamente, el conocimiento y las tecnologías que recibe.

Por otro lado, la literatura sobre economía de la innovación ha evolucionado en los últimos años y ha tratado de incorporar de manera explícita en su análisis a las instituciones para explicar los procesos de innovación (véase, por 
ejemplo, Nelson y Sampat, 2001; Hage, 2006; Hollingsworth, 2006). La economía institucional -y en particular la perspectiva de cambio institucional (CI)considera categorías de análisis que han sido profundamente desarrolladas por la economía evolutiva para explicar el rol de las instituciones y su influencia en los procesos de interacción y en el comportamiento de los actores.

En este sentido, los patrones de comportamiento se conciben en términos conductuales, pues se explican a través de procesos de razonamiento, inducción y aprendizaje tanto individual como colectivo enmarcados en hábitos, normas, reglas, valores y rutinas. ${ }^{3}$ En este proceso conductual el conocimiento y el aprendizaje son conceptos centrales en la explicación de la recreación de nuevo conocimiento, en el desarrollo de capacidades de innovación en los procesos de transformación de los microfundamentos de las instituciones.

Una de las definiciones de conocimiento que mejor reflejan su papel en un entorno organizacional e institucional determinado es la que ofrecen Davenport y Prusak (1998): "una combinación fluida de experiencias, valores, información contextual e ideas expertas que proveen una estructura para evaluar e incorporar nuevas experiencias e información, se origina y es aplicado en la mente de los conocedores. En las organizaciones a menudo se incorpora en rutinas organizacionales, procesos, prácticas y normas, y no sólo en documentos”. Por ello, los procesos de transmisión deben apoyarse en un adecuado sistema de gestión que permita transformar la información generada por diversas fuentes externas e internas en nuevo conocimiento.

La diferencia entre conocimiento tácito y codificado es fundamental para explicar las formas de interacción en los procesos de innovación. Ambos tipos de conocimiento son creados por las personas, pero el primero es heurístico, subjetivo e interno, se aprende a través de ejemplos empíricos (Arrow, 1962), de la experiencia y de la práctica (Rosenberg, 1982), es personal y de contexto específico (Polanyi, 2009; Nonaka y Takeuchi, 1995), no es fácil de comunicar (transferir) y formalizar.

A su vez, el conocimiento codificado se puede transmitir utilizando el lenguaje formal y sistemático (Senker y Faulkner, 1996). La naturaleza del sector y el entorno institucional son determinantes de la manera en que las organizaciones acceden al conocimiento, y la capacidad tecnológica y organizacional de

${ }^{3}$ Según Hodgson (2007), las rutinas son metahábitos organizacionales que se diseminan entre las organizaciones en un medio ambiente institucional. 
éstas influye en el grado de acumulación, lo cual se puede lograr a través de actividades de aprendizaje individual, grupal y organizacional. ${ }^{4}$

El aprendizaje organizacional también juega un papel crucial en este panorama. Por un lado, los individuos adquieren destrezas técnicas y conocimiento a través de diversos procesos productivos y organizacionales, lo que en ocasiones depende exclusivamente de la experiencia, como el aprender haciendo (learning by doing) (Arrow, 1962; Bell y Pavitt, 1995) o el aprender por el uso (learning by using) (Rosenberg, 1982), pero también depende de los procesos de socialización del conocimiento (learning by interacting) (Lundvall, 1992), pues a través de éstos las organizaciones aprenden. La manera como cada organización acumula y genera nuevo conocimiento depende de las actividades de aprendizaje y de los mecanismos de absorción, asimilación y explotación que haya desarrollado previamente.

\section{Modos de coordinación para un análisis de cambio microinstitucional}

Recientemente, académicos y tomadores de decisiones han expresado su preocupación sobre los procesos de cambio en instituciones que se han desarrollado dentro del modelo económico actual.

A partir de un severo cuestionamiento de las grandes disparidades en la asignación, distribución y explotación de recursos, se ha analizado desde distintos campos de las ciencias sociales, tanto en el marco normativo como en el de los hechos, el papel de las instituciones en este modelo. Particularmente, los marcos teóricos de la economía evolutiva e institucional han coincidido en el desarrollo de categorías conceptuales y analíticas para analizar el efecto que tienen las instituciones y su influencia en el desarrollo y desempeño (económico y social) de organizaciones (de mercado y no-mercado) que emergen dentro de este modelo.

En una reflexión en retrospectiva, este esfuerzo se ha hecho desde los inicios de la teoría económica. Veblen (2007) y Commons (2003) son dos de los primeros economistas que explicaron cómo surgen las instituciones, cómo inciden en las elecciones de los actores económicos y cómo éstos se desempeñan en

\footnotetext{
${ }^{4}$ Los incentivos encuadrados en el marco institucional dirigen el proceso de aprendizaje mediante la enseñanza-práctica y el desarrollo del conocimiento tácito, necesario para la toma de decisiones. Normas institucionales diferentes producirán incentivos diferentes para el conocimiento tácito (North, 1990).
} 
un entorno económico e institucional determinado. ${ }^{5}$ No obstante, por mucho tiempo las instituciones se han considerado exógenas en los modelos explicativos de maximización en la teoría neoclásica y estáticas en la economía institucional. ${ }^{6}$

Estas expresiones se encuentran en las diversas definiciones de institución. Por ejemplo, Schotter (1981) la considera como la regularidad general en el comportamiento social, mientras que para North (1990) son las reglas del juego que constriñen y moldean la interacción humana. Varios autores coinciden en que las instituciones constituyen el marco en el que tienen lugar las interacciones y reducen la incertidumbre al proporcionar estructuras de interacción.

Si bien el concepto de institución se ha ampliado en las últimas décadas, la perspectiva teórica del CI ha tratado de explicar tanto los procesos de su creación como de cambio y coevolución en distintos niveles. A la luz de este enfque, las instituciones son consideras endógenas y en coevolución.

Diversos conceptos evolutivos y teorías se han incorporado directa o indirectamente a la base del análisis para explicar las instituciones y sus dinámicas. Por ejemplo, desde la visión de North, el cr conforma el modo en que las sociedades evolucionan a lo largo del tiempo, "explica la forma en que el pasado influye en el presente y en el futuro, la manera en que el cambio institucional incremental afecta la elección establecida en un cierto momento" (North, 1990, p. 13), y es la clave para entender el cambio histórico. ${ }^{7}$ Esta definición incorpora implícitamente el concepto de "dependencia" (path dependence) para explicar los procesos de cambio a nivel macroinstitucional. ${ }^{8}$ Desde la perspectiva de la dependencia, se asume que los actores están totalmente determinados por las elecciones hechas en el pasado o por las instituciones/organizaciones a las cuales se incorporan y que los individuos realizan sus elecciones considerando no sola-

\footnotetext{
${ }^{5}$ Si bien los economistas clásicos como Smith (1983) y Mill (1998) fueron pioneros en explicar los procesos del intercambio económico a partir de la noción de instituciones, T. Veblen (18571929) fue uno de los fundadores del institucionalismo económico moderno al criticar a la economía dominante por no comprender la influencia de los valores, tradiciones, leyes y la cultura en los procesos de asignación de recursos y maximización de beneficios.

${ }^{6} \mathrm{Si}$ bien una parte del análisis económico ha sido evolutivo e institucional, ni la tecnología ni las instituciones se incorporaron endógenamente o formalmente a los axiomas explicativos de la maximización en la teoría económica neoclásica.

${ }^{7}$ Para North (1990), el CI consiste en ajustes marginales al conjunto de reglas, normas y cumplimiento del marco institucional.

${ }^{8}$ La dependencia (path dependence) se define como un continuo del modo dominante que sustituye a la coordinación del mercado a través de un número de sectores industriales, categoría ampliamente analizada por David (1985), Arthur (1994) y Nelson y Winter (2002).
} 
mente las acciones previas sino también los costos de cambiar esas elecciones (Hollingsworth, 2006).

Desde esta perspectiva, Hage (2006) define el Cr como un cambio dominante en los modos de coordinación en una sociedad (i.e., un cambio de los arreglos institucionales). Entre los modos de coordinación de no-mercado, Hage incluye a las redes interorganizacionales.

Los modos de coordinación entre los diferentes actores establecen instituciones para asegurar los beneficios mediante la formación de distintas configuraciones que facilitan las relaciones de complementariedad y de coordinación entre las instituciones, así como también la emergencia de nuevas formas de autocoordinación (Ayala, 2001). Estos modos varían entre sectores industriales (y niveles de análisis). Según Hage (2006), el sector servicios y las industrias de producción en masa se caracterizan por tener modos de coordinación institucional dominantes (con una jerarquía corporativa a través de la coordinación de los precios, salarios, oferta), pero son menos dominantes en los sectores basados en la ciencia (nuevos y pequeños).

Una de las preguntas actuales es cómo cambian las instituciones inmersas en un entorno socioeconómico complejo, dinámico y en constante cambio tecnológico, y cómo tal entorno recrea y fomenta la creación de nuevos modos de coordinación y nuevas instituciones. North (1990) argumenta, a partir de un enfoque de la elección racional, que las premisas del cambio son los actores y la variación de precios relativos.

Campbell (2004, 2007 y 2009) propone una teoría del CI centrada en los actores, los cuales construyen y modifican las instituciones para alcanzar sus intereses. A partir de un nivel de análisis microinstitucional, Campbell (2004, 2007 y 2009) desarrolla una estructura teórico-conceptual para fundamentar el análisis macroinstitucional del CI.

Para este autor, este cambio puede ser intencional o no intencional y el que importa es el primero, definido como "un esfuerzo deliberado para reorganizarse o modificar las instituciones existentes", es decir, 1) las reglas formales e informales, 2) los mecanismos de supervisión y ejecución, y 3) los sistemas de significado que definen el contexto en el cual operan los actores e interactúan entre sí. "Las instituciones son acuerdos que nacen de la lucha y la negociación [...] por ello reflejan los recursos y el poder de las personas que las han creado [...] Una vez creadas las instituciones afectan la distribución de recursos y poder entre las personas vinculadas con la institución [...]" (Campbell, 2009, pp. 14 y 19). 
Siguiendo a Campbell (2009), las categorías centrales del CI son los actores, el cambio y la adaptación. Desde esta óptica, los actores perciben problemas que requieren soluciones institucionales y son los emprendedores los que sugieren cómo recombinar elementos institucionales en formas innovadoras, pero aún se sabe poco sobre la manera en que los actores desarrollan estas percepciones.

El CI implica un proceso de combinación y recombinación de elementos organizacionales e institucionales ya existentes, proceso ampliamente explicado desde un enfoque distinto por autores como Levi-Strauss (1966) y Douglas (1986) y conocido como "bricolaje" (término utilizado en la literatura sobre entrepreneurship) e incorporado en el análisis microinstitucional de Campbell. A esta categoría de cambio se asocia la de estabilidad. ${ }^{9}$ Por un lado, una transformación revolucionaria supone cambios simultáneos en la mayor parte o en todas las dimensiones de una institución, por otro, una evolutiva supone cambios sólo en algunas de esas dimensiones. La teoría de la innovación ha explicado muy bien estas diferencias. A su vez,, la estabilidad implica la ausencia de cambio en la mayoría de las dimensiones. Así, el CI es un continuo que va de la estabilidad, pasando por el cambio evolutivo en el tramo central, al más revolucionario en el otro extremo.

Eventualmente, en los entornos institucionales locales se incluyen elementos nuevos para ellos pero ya existentes en otros entornos, que se modifican y ajustan al ser incorporados. A este proceso, Campbell (2009) lo denomina adaptación.

Si bien la economía institucional ha puesto atención en los patrones históricos para explicar las instituciones, ha tenido limitaciones para trasladar esto a un modelo dinámico (Van den Bergh y Stagl, 2003). La preocupación de Campbell por desarrollar una estructura conceptual del CI centrada en los actores valida la vieja inquietud de incorporar al individuo en el centro de los procesos de creación, evolución y dinámica de las instituciones. Al centrar su análisis en los actores, este autor toma en cuenta de manera implícita la estructura cognitiva de los actores en esos procesos.

De este modo, sin entrar en la vieja discusión determinista sobre individuos e instituciones y considerando la variación de las estructuras cognitivas de los actores, es necesario tomar en cuenta los procesos de aprendizaje, transfor-

\footnotetext{
9 "La estabilidad se logra por la preservación de reglas [formales e informales eficientes] y el cambio aparece cuando los actores deciden cambiar estas reglas" (Julve, Prats y Comas, 2009), tanto a nivel micro-, meso- y macroinstitucional.
} 
mación del conocimiento y cambio de normas y reglas. Para algunos autores de la escuela neoinstitucionalista, como Hodgson (2007), Hage (2006) o Hollingsworth (2006), es posible percibir un consenso respecto a que las estructuras sociales más altas emergen desde las interacciones complejas entre los individuos, de sus hábitos y conocimiento acumulados. ${ }^{10}$

Desde otras perspectivas evolutivas que aportan a la construcción teórica del CI a partir del comportamiento individual, la sociobiología, por ejemplo, incluye la selección de parentesco, las aptitudes inclusivas y el altruismo recíproco. ${ }^{11}$ La psicología evolutiva considera que los humanos genéticamente se comportan aún como lo hacían los cazadores-recolectores del Pleistoceno y, por tanto, los individuos asumen estrategias de comportamiento de manera automática. La idea que plantean estos autores es que hay una evolución cultural y biológica con interacciones positivas y negativas que definen una coevolución genético-cultural (Van den Bergh y Stagl, 2003).

En este sentido, la construcción o recombinación de instituciones contribuye a la estabilidad, resistencia, adaptabilidad y velocidad de la innovación. Y las organizaciones económicas se adaptan rápidamente para cambiar sus circunstancias, lo cual contribuye, según Van den Bergh y Stagl (2003), a un incremento acelerado del ritmo de evolución económico-cultural.

\section{Metodología}

La estrategia de investigación de este trabajo se basa en un estudio de caso múltiple-exploratorio. Los casos son las unidades médicas de salud, la unidad de análisis se refiere a los proyectos de telemedicina.

El tipo de información analizada es cualitativa y los resultados presentados se sustentan en información recopilada a través de 14 entrevistas a profundidad a líderes de proyecto, técnicos y médicos de unidades del sector salud público (pertenecientes a la Secretaría de Salud) en los estados de Guerrero, Oaxaca y Chiapas, entre junio de 2011 y enero de 2012. Más de un actor fue entrevistado en más de una ocasión. Se abordaron diversos temas clave con diferentes

\footnotetext{
${ }^{10}$ Para el individualismo metodológico de Hodgson (2007), las instituciones surgen a partir de las interacciones individuales en entornos socioeconómicos que moldean los objetivos o preferencias de los individuos.

${ }^{11}$ De alguna manera, la teoría evolutiva ha incorporado los axiomas de racionalidad individual en la cooperación económica (transacciones) y el altruismo mediante la evaluación de costos $\mathrm{y}$ beneficios.
} 
entrevistados pertenecientes a un mismo proyecto, como mecanismo de validación y confiabilidad de la información.

En el cuadro 1 se muestran las categorías analíticas utilizadas y las formas de evaluación de cada una.

Cuadro 1. Operacionalización de las categorías analíticas

\begin{tabular}{|l|l|}
\hline \multicolumn{1}{|c|}{ Categoría } & \multicolumn{1}{c|}{ Forma de evaluación } \\
\hline $\begin{array}{l}\text { Identificación de } \\
\text { conocimiento }\end{array}$ & $\begin{array}{l}\text { A través de la importancia que tienen las diversas fuentes } \\
\text { de información y la frecuencia con la que adoptan nuevas } \\
\text { tecnologías. }\end{array}$ \\
\hline $\begin{array}{l}\text { Internalización de } \\
\text { conocimiento }\end{array}$ & $\begin{array}{l}\text { Mediante las actividades de aprendizaje (capacitación, foros } \\
\text { de discusión, asistencia a congresos, etcétera) y formas de } \\
\text { solución de problemas. }\end{array}$ \\
\hline Difusión de conocimiento & $\begin{array}{l}\text { A través de los procesos de intercambio y coordinación de } \\
\text { experiencias. }\end{array}$ \\
\hline Explotación de conocimiento & $\begin{array}{l}\text { En razón de la percepción que tienen los profesionales del } \\
\text { ramo sobre los efectos de la telemedicina en la mejora de los } \\
\text { servicios de salud. }\end{array}$ \\
\hline $\begin{array}{l}\text { Coordinación } \\
\text { intraorganizacional }\end{array}$ & $\begin{array}{l}\text { Por medio de las formas de trabajo coordinado entre los } \\
\text { actores para la toma de decisiones a partir del uso de la } \\
\text { telemedicina. }\end{array}$ \\
\hline
\end{tabular}

Fuente: Elaboración propia.

Sobre la base de lo anterior, los conceptos centrales que ayudan a explicar el comportamiento de los actores (individuos, grupos, organizaciones, sectores) en un marco institucional son el conocimiento, el aprendizaje colaborativo y los modos de coordinación. Este marco institucional da forma a la dirección de la adquisición del conocimiento (North, 1990), es decir, al diseño de los procesos de aprendizaje, en el que los agentes del cambio son los emprendedores institucionales considerados por Campbell (2009) que se involucran en procesos de aprendizaje y recreación de cúmulos de conocimiento.

\section{Retos Del SeCtor SALUd EN MÉXICO}

El sistema de salud en México enfrenta retos importantes, principalmente de inversión en infraestructura, tecnología y recursos humanos especializados, así como de ampliación de la cobertura, mejor eficiencia, equidad y reestructuración tanto organizacional como institucional. En el Informe Mundial de la Salud 2000 (OMS, 2000) se argumentó que los países en desarrollo en general tienen sistemas de salud burocráticos, centralizados y jerárquicos, mal administrados y fragmen- 
tados en diversos programas verticales, y a menudo son miopes en la definición de estrategias de atención de la salud de largo plazo.

Aunque los tomadores de decisiones han diseñado e implementado estrategias para enfrentar esos retos, principalmente la ampliación de la cobertura, a esto se suma una dinámica de transformación epidemiológica y geográfica superior a la capacidad de respuesta de los actores del sistema. El cambio demográfico implica un cambio en la prevalencia de enfermedades por tipo de población, de tal forma que es urgente la implementación de estrategias de atención a la salud, particularmente en México, pues su población se vuelve cada vez más vieja.

\section{Gastos en salud}

Una de las tareas fundamentales de los sistemas de salud es alcanzar la cobertura universal de seguridad social. En México, este objetivo se estructura con cinco actores principales que representan diferentes esquemas de financiamiento. El primero es el Instituto Mexicano del Seguro Social (IMSs), financiado por un esquema de prepago de los trabajadores formales del sector privado. El segundo es el Instituto de Seguridad y Servicios Sociales de los Trabajadores del Estado (ISSSTE), también financiado por un esquema de prepago, pero de los empleados gubernamentales; la Secretaría de la Defensa Nacional (SEDENA) también utiliza este esquema. El tercero es la Secretaría de Salud (ss), que obtiene recursos mediante cuotas de recuperación de la población abierta. El cuarto actor es el subsistema privado (SP), que se financia con la venta de seguros médicos a la población abierta. Un nuevo actor del sistema de salud mexicano es el Seguro Popular, el cual ofrece paquetes de servicios de salud básicos financiándose con cuotas de recuperación de la población abierta. De acuerdo con Molina y Sánchez (2009), los primeros dos actores atienden a $50 \%$ de la población y los otros tres podrían atender al resto.

Aunado a la baja cobertura y una mala educación en materia de sanidad e higiene (Mosqueira y Correa, 2009), el gasto total en salud con respecto al producto interno bruto (PIB), en 2008, de México fue de 5.1\%, contra 7.2\% de Brasil y $13.4 \%$ de Estados Unidos.

Como se muestra en el cuadro 2 , en ese mismo año, $53.1 \%$ del financiamiento del sistema de salud provino principalmente de los hogares; el resto, del sector público. Pero, como mencionan Molina y Sánchez (2009), lo importante es la carga de ese gasto para los hogares: del total del financiamiento que provie- 
Cuadro 2. Gastos en salud: total, público y privado. Países seleccionados, 2000 y 2008 (porcentajes)

\begin{tabular}{|l|c|c|c|c|c|c|}
\hline \multirow{2}{*}{ País } & \multicolumn{2}{|c|}{$\begin{array}{c}\text { Gasto total en salud } \\
\text { como porcentaje del PIB }\end{array}$} & \multicolumn{2}{c|}{$\begin{array}{c}\text { Gasto público en salud } \\
\text { como porcentaje del } \\
\text { gasto total en salud }\end{array}$} & \multicolumn{2}{c|}{$\begin{array}{c}\text { Gasto privado en salud } \\
\text { como porcentaje del } \\
\text { gasto total en salud }\end{array}$} \\
\cline { 2 - 7 } & 2000 & 2008 & 2000 & 2008 & 2000 & 2008 \\
\hline México & 5.1 & 5.9 & 46.6 & 46.9 & 53.4 & 53.1 \\
\hline Brasil & 7.2 & 8.4 & 40.3 & 44.0 & 59.7 & 56.0 \\
\hline Estados Unidos & 13.4 & 15.2 & 43.2 & 47.8 & 56.8 & 52.2 \\
\hline
\end{tabular}

Fuente: oms (2011).

ne de los hogares, $94 \%$ se utiliza para pagos directos a los proveedores de servicios de salud, $5 \%$ para cubrir las primas de los seguros privados y $1 \%$ para cubrir cuotas de recuperación. Como bien señalan dichos autores, este esquema es una forma regresiva de financiamiento, pues los hogares prácticamente están financiando al sistema, en particular los de menores ingresos.

\section{Cambio demográfico y epidemiológico}

Nuevos retos demográficos y epidemiológicos se suman a la baja capacidad de financiamiento en salud por parte del Estado y a la baja capacidad de gasto de los hogares pobres para cubrir sus necesidades de salud. En los últimos años, México ha enfrentado un proceso de envejecimiento más rápido que el descenso de la mortalidad (la tasa bruta de mortalidad pasó de 5.6 por mil en 1990 a 5 por mil en 2010), sumado a un aumento de la esperanza de vida (la cual pasó de un promedio de 70.6 en 1990 a 75.4 años en 2010) (CONAPO, 2012) y a un cambio en la pirámide demográfica (la proporción de la población de entre 0 y 14 años pasó de $34.1 \%$ en 2000 a $29.3 \%$ en 2010 y será de $24.3 \%$ en 2020 , mientras que la población de 60 años y más pasó de $4.7 \%$ en 2000 a $6.2 \%$ en 2010 y será de $8.5 \%$ en 2020). Los retos para el sistema de salud son más que obvios.

La prevalencia de enfermedades también está cambiando. Entre 1990 y 2004, las enfermedades infectocontagiosas, como las respiratorias, disminuyeron (a excepción de algunos estados como Chiapas, donde aún son frecuentes), ${ }^{12}$ y está aumentando la prevalencia de enfermedades crónico-degenerativas, entre

${ }^{12}$ La prevalencia de estas enfermedades está asociada a las condiciones de pobreza y a la falta de infraestructura adecuada de servicios de salud (Molina y Sánchez, 2009). 
ellas las cardiovasculares y la diabetes, las cuales producen más de $55 \%$ de las muertes anuales (Molina y Sánchez, 2009).

Una pregunta obligada es cómo atender a una población con una esperanza de vida cada vez mayor que presenta nuevas enfermedades y nuevos retos para el sector salud. Sin duda, la prevención es fundamental para disminuir los riesgos de salud y los costos de atención pero, según los expertos, no hay una política de prevención y la población no muestra una cultura al respecto, por lo tanto, seguirán vigentes los retos financieros, institucionales, tecnológicos, organizacionales, etcétera, para el diagnóstico, tratamiento, monitoreo y seguimiento de enfermedades de mayor prevalencia relativa (diabetes y obesidad, entre otras).

\section{Difusión de las tecnologías de la información y la comunicación (TIC) y dinámica tecnológica}

México ha avanzado en algunos indicadores de salud, pero se ha estancado en otros. Por ejemplo, según datos de la Organización Mundial de la Salud (oms, 2011), en 2008 tenía 28.9 médicos por cada diez mil habitantes, contra 26.7 de Estados Unidos; 16 camas por cada diez mil habitantes, contra 31 en Estados Unidos, y 0.9 unidades de radioterapia por cada millón de habitantes, contra 11.8 en Estados Unidos.

En el cuadro 3 se observa una agudización del retraso tecnológico de México, particularmente en la adquisición de tomógrafos y unidades de resonancia magnética, y no sólo por la escasez de esos equipos médicos sino por la capacidad casi nula para diseñarlos y desarrollarlos internamente.

Cuadro 3. Principales tecnologías médicas por millón de habitantes

\begin{tabular}{|l|c|c|c|c|}
\hline \multirow{2}{*}{ País } & \multicolumn{2}{|c|}{ Tomógrafos } & \multicolumn{2}{c|}{$\begin{array}{c}\text { Unidades de resonancia } \\
\text { magnética }\end{array}$} \\
\cline { 2 - 5 } & 2001 & 2009 & 2001 & 2009 \\
\hline México & 2.51 & 4.34 & 1.1 & 1.94 \\
\hline Israel & 6.52 & 9.35 & 1.4 & 1.87 \\
\hline Estados Unidos & 28.87 & $34.29 *$ & 20.1 & $25.91^{*}$ \\
\hline
\end{tabular}

*Dato para 2007.

Fuente: OECD. (2011).

Una de las premisas fundamentales del desarrollo es aumentar el bienestar socioeconómico de la población, no solamente con mejores productos, sino también con mejores servicios, entre ellos la atención a la salud, la cual debe ser 
de calidad, eficiente y equitativa. Desde la perspectiva de la economía de la innovación, un requisito consiste en que la calidad de los servicios de atención a la salud se debería de incrementar con el progreso tecnológico.

La incorporación de nuevas tecnologías, la creación de nuevo conocimiento y la recreación de cúmulos de conocimiento ya existentes están presentes en la práctica médica desde sus inicios, sin embargo, el cambio tecnológico vertiginoso de las últimas cuatro décadas ha transformado las estructuras socioeconómicas de todos los sistemas de salud, unos en mayor medida que otros.

Ante la emergencia de las TIC en la década de los setenta, el sector salud no escapa a la transversalidad tecnológica de éstas. A partir de ellas se han creado nuevos productos, procesos y servicios (como la teleeducación, la teleconsulta y el expediente clínico electrónico), así como nuevas áreas o departamentos (unidades de tecnologías de la información, cómputo, etcétera). La telemedicina es una de las áreas fundamentales del sector salud que incorpora las TIC en sus procesos; es una práctica médica que nace hace casi un siglo directamente relacionada con las telecomunicaciones. El uso del teléfono es la primera forma de teleconsulta y más tarde las tecnologías de la información (TI) le dan un nuevo giro a las consultas a distancia, redefiniendo la telemedicina.

En la literatura médica hay diversas definiciones de telemedicina y todas están asociadas al uso de las TIC para apoyar a los servicios médicos. Adicionalmente a esta idea general, autores como Rooney (1999) ponen el acento conceptual en la distancia geográfica, mientras que Fong, Fong y Li (2009) enfatizaron las diversas aplicaciones que apoyan la transmisión de información médica. En este trabajo, la telemedicina es una tecnología social asociada al uso de TIC (hardware, software, redes, comunicaciones) para la transferencia y difusión de datos, información y conocimiento (en forma de sonido, imagen, texto) relacionados con la salud entre profesionales de la medicina y usuarios de los servicios de salud.

En varios estudios se ha evidenciado el potencial de la telemedicina como una tecnología social que permite incrementar el acceso a los servicios de salud y la calidad de éstos a bajo costo. A pesar de ello y de los avances en la capacidad y disponibilidad de las TIC, la tasa de utilización de proyectos de telemedicina instalados ha sido, y permanece, baja (Paul, 2006).

En los párrafos anteriores se muestra una ventana de amplias posibilidades de investigación desde diferentes perspectivas teóricas. Desde la óptica de la economía de la innovación, y considerando que la tecnología incorpora diferentes cúmulos de información y conocimiento, hay poca evidencia de cómo las 
organizaciones médicas diseñan, desarrollan e implementan procesos de asimilación, internalización, difusión y explotación del conocimiento.

Al mismo tiempo, si bien hay una amplia literatura sobre economía de la salud que ha explorado el desempeño en el sector salud, Molina y Sánchez (2009) evidencian la escasa regulación en el sector, mientras que Molina y Carbajal (2009) han identificado problemas de información asimétrica que afectan la toma de decisiones, además de, como se mostrará aquí, los problemas de coordinación y cambio institucional.

\section{RESULTADOS. LA TELEMEDICINA Y SUS EFECTOS EN LOS SERVICIOS DE ATENCIÓN A LA SALUD: INNOVACIÓN Y CAMBIO MICROINSTITUCIONAL}

Este apartado se basa en el análisis de información obtenida en entrevistas a profundidad durante el trabajo de campo exploratorio sobre los proyectos de telemedicina en unidades médicas del sector salud, en las entidades de Oaxaca, Chiapas y Guerrero.

\section{Adopción de nuevas tecnologías y cambios en las percepciones y comportamiento de los actores}

Con la premisa de que la calidad de los servicios de salud se incrementa con el progreso tecnológico, las tecnologías médicas como la telemedicina también se consideran herramientas necesarias en términos de democratización y equidad en la prestación de tales servicios.

El sector salud absorbe constantemente tecnología tanto diagnóstica como terapéutica y es uno de los de mayor dinamismo en la transformación y generación de conocimiento. Sin duda, la incorporación de TI en la práctica médica tiene por lo menos un siglo, pero la forma en que se concibe actualmente a las TIC y su adopción en diversos sectores, como el de salud, es relativamente reciente.

En general, a pesar de las bondades de la aplicación de las TIC a diversos procesos productivos, organizacionales, tecnológicos y científicos, algunos sectores, por ejemplo, el de salud público, han mostrado diversos problemas en su absorción. Particularmente, en las unidades médicas analizadas, los médicos de edad avanzada han hecho evidente su resistencia al uso de la telemedicina. Bashshur, Reardo y Shannon (1997) también encontraron esta resistencia como uno de los principales factores que limitan la implementación adecuada de la telemedicina. 
La puesta en práctica de la telemedicina implica un cambio en las estructuras organizacionales o creación de nuevas áreas, como la de informática. A micronivel, este rediseño implica un cambio de paradigma organizacional en la adopción de nuevas tecnologías. Este argumento tiene diferentes causas. Por un lado, el ámbito generacional tiene un peso importante en la manera en que los médicos perciben las tecnologías en los procesos de toma de decisiones de su profesión; los de generaciones más antiguas muestran una relativamente alta aversión a adoptar las TIC en los procesos de atención médica y, por tanto, al cambio en la toma de decisiones.

Particularmente, algunos médicos mencionan que la pérdida de contacto directo con los pacientes y la atención a éstos a través de dispositivos digitales es una limitación en el uso de la telemedicina. Para estas generaciones, valerse de este recurso implica no sólo un cambio de percepciones en la práctica médica con el uso de las TIC, sino también un cambio en el modelo de atención médica que se ha arraigado con el tiempo. ¿Cómo cambiar el modelo casi medieval de atención a la salud aún vigente y que ha hechado raíces en los patrones de comportamiento de la comunidad médica? Sin embargo, a pesar de una amplia difusión de las TIC (telefonía móvil, Internet, gadgets, etcétera), en los ambientes de educación formal los médicos más jóvenes también han mostrado una limitación a la adopción y explotación de la telemedicina.

Si bien el uso de ésta implica un cambio en la percepción y en los patrones de comportamiento de los actores involucrados, tales modificaciones graduales se realizan a través de los esfuerzos de los médicos para rediseñar sus estructuras cognitivas en los procesos de internalización y explotación de esa tecnología. Estos cambios en las percepciones y en los patrones de comportamiento se explican por los modelos de atención que involucran diversos mecanismos de aprendizaje, difusión y transformación del conocimiento, así como distintos modos de coordinación y toma de decisiones.

\section{Aprendizaje y difusión de conocimiento en dos modelos de atención médica}

En un primer modelo de atención médica, la telemedicina no se internaliza y predomina la relación médico-paciente en las diferentes etapas de una enfermedad (diagnóstico, tratamiento, monitoreo y seguimiento). Este patrón se repite tantas veces como la gravedad de la enfermedad lo requiera, es decir, no importa si el padecimiento es una gripe común o un cáncer. Por supuesto, las capacidades médicas serán proporcionales a la complejidad de la enfermedad y los procesos de 
aprendizaje basados en la educación formal y experiencia personal serán graduales conforme el tipo de especialización. Los procesos formales de difusión y adquisición de conocimiento entre la comunidad médica se restringen a la asistencia a congresos, seminarios, coloquios, etcétera, y a la experiencia adquirida a través del ejercicio de la práctica médica. En este modelo los modos de coordinación son jerárquicos, con una estructura vertical entre los médicos de mayor y de menor especialización.

Un segundo modelo de atención, que implica la internalización de la telemedicina, asegura la participación coordinada de dos o más doctores en la atención de un paciente, particularmente en enfermedades de mediana o alta especialidad que no pueden ser atendidas por médicos generales. Las capacidades de los actores son diferenciadas pero coordinadas por un doctor ubicado en una unidad médica de baja especialidad o de primer nivel. Es decir, la triangulación de información entre el paciente y el especialista se da mediante la coordinación de un médico general.

A partir del principio de que este modelo tiene como objetivo atender a la población que no puede acceder a servicios de alta especialidad por su condición socioeconómica o porque la geografía limita su traslado, esta interacción a distancia entre un especialista (ubicado en un hospital de especialidades) y el paciente se da a través de un médico general o practicante (ubicado en una unidad clínica de la localidad rural o indígena). En este modelo el paciente ofrece información sobre su enfermedad que es decodificada por el médico general y transferida al especialista mediante la telemedicina. Éste realiza un diagnóstico de la enfermedad y transfiere conocimiento sobre su tratamiento, monitoreo y seguimiento al médico general, quien, a su vez, la decodifica en un lenguaje que comprenda el paciente.

El médico general efectúa procesos de aprendizaje informal a través de la telemedicina, pues adquiere conocimiento codificado y tácito del especialista, por supuesto, de manera parcial. (En el modelo anterior, la adquisición de conocimiento especializado, aun parcial, sería más costoso). Al mismo tiempo, el especialista transfiere su conocimiento al médico general, y no al paciente, a través de la telemedicina, lo cual implica un proceso importante de transformación del conocimiento tácito y codificado a partir de una estructura cognitiva básica. Esta tecnología permite, además, la conformación de foros de discusión instantánea y capacitación informal.

En este modelo de atención basado en la telemedicina, los modos de coordinación son menos jerárquicos y con una estructura casi horizontal entre 
los actores (médicos especialista, general y paciente); asimismo, hay procesos de colaboración al seno de la comunidad médica, los cuales pueden influir en una mejor atención al paciente.-

En este sentido, la difusión de conocimiento entre los médicos a través de la telemedicina implica una mayor socialización del conocimiento tácito y una estrategia de aprendizaje. Si bien hay estándares y procedimientos rigurosamente regulados en la práctica médica, hay importantes cúmulos de conocimiento que se mantienen tácitos y una nueva forma de transmitirlos es por medio de la teleconsulta. ${ }^{13}$

Los cambios en el modelo de atención (cambios en los patrones de comportamiento de los actores y en sus percepciones) deben operar en los primeros niveles de atención. En las unidades médicas analizadas se encontró una relativa resistencia a la implementación y uso de la telemedicina, una resistencia que puede agudizar los problemas de saturación de la demanda en las unidades médicas de tercer nivel o de alta especialidad, con lo que el sistema será insostenible en el mediano plazo para atender a la población que requiere cada vez más servicios de salud por enfermedades crónicas y degenerativas. Ante el cambio demográfico y epidemiológico, los sistemas de salud no tienen capacidad para soportar la carga financiera de una nueva demanda de sus servicios, razón por la cual algunos actores enfatizan la necesidad de fomentar el uso de las nuevas tecnologías y su adecuada explotación en las unidades médicas de primer nivel.

Específicamente, la telemedicina tiene la capacidad para acelerar los cambios de manera gradual en el control de las etapas iniciales de ciertos padecimientos (enfermedades coronarias, diabetes, hipertensión, etcétera). Pero por su pertinencia, en el tercer nivel, es decir, los hospitales de mayor capacidad resolutiva, también se tendrán que adoptar modelos de atención a distancia a través de la telemedicina (seguimiento y monitoreo domiciliario -teleasistencia-), primero por los costos menores (tanto para los hospitales como para los usuarios) que implica atender estas actividades médicas con la capacidad instalada actual $\mathrm{y}$, segundo, por la apropiación del paciente de su corresponsabilidad en su salud.

\footnotetext{
${ }^{13}$ Algunos médicos sostienen que sus colegas tienen limitaciones para compartir el conocimiento tácito, de manera que la posibilidad de retransformarlo también es limitada. Si bien las TI son un artefacto que fomenta esa socialización, dada la resistencia a compartir el conocimiento de manera presencial, no hay motivo para pensar que lo harían a través de estas tecnologías. Esta paradoja es interesante si se consideran casos (como en la psiquiatría) en los que existe evidencia de procesos de socialización del conocimiento tácito.
} 


\section{Modos de coordinación como elementos de la dinámica de cambio}

Las nuevas tecnologías como la telemedicina empiezan a cambiar los modelos de atención de la práctica médica. Por un lado, hay un cambio en la percepción que tiene los médicos de la importancia de las TIC en los procesos de rediseño de los modelos de atención y en la necesidad de compartir conocimiento con doctores de menor especialidad. Por otro lado, estos cambios de comportamiento hacia la telemedicina y hacia el tratamiento de pacientes a través de nuevas tecnologías implica un cambio en los modos de coordinación intraorganizacional entre unidades médicas. Los modos de coordinación están delimitados por la capacidad de los médicos para socializar el conocimiento tácito a través de las nuevas tecnologías, por el modelo particular de atención según el tipo de unidad y el tipo de enfermedades de mayor prevalencia según el área geográfica.

La estructura institucional del sector está caracterizada por códigos de ética, normas rigurosamente reguladas por organizaciones tanto nacionales como extranjeras, procedimientos unificados, símbolos o lenguaje estandarizado, entre otros elementos, de tal manera que las rutinas médicas son homogéneas en los segmentos de alta especialidad y su transferencia no es limitada por la telemedicina, por el contrario, se reproducen con mayor dinamismo. En este sentido, un actor-interfaz facilita la transmisión de hábitos, normas, reglas, rutinas y convenciones, pero cuando las organizaciones se involucran en procesos de exploración, o donde los entornos son dinámicos y complejos y predomina el conocimiento tácito, la reproducibilidad de estos elementos institucionales es sumamente compleja.

\section{Explotación del conocimiento y efectos de la telemedicina en los servicios de atención a la salud}

Los efectos de la telemedicina son diferenciados. Si bien los resultados del trabajo de campo son exploratorios, se encontraron los siguientes efectos: los entrevistados coinciden en que la telemedicina influye en los procesos de diagnóstico y monitoreo de las enfermedades, ya que reduce de manera importante sus costos tanto para las unidades médicas como para los usuarios, incrementa la calidad y reduce los tiempos de la atención, pues los pacientes no tienen que trasladarse a las zonas urbanas, de forma que influye en una reducción del gasto en transporte para la gente de escasos recursos. Estos resultados serán validados estadísticamente en un estudio posterior (mediante una encuesta entre líderes de 
proyecto de telemedicina y nuevas tecnologías, y pacientes). La prueba piloto realizada con líderes de proyecto coincide con estos resultados cualitativos basados en estudios de caso.

Otro efecto evidenciado es una mejora gradual en el uso de los recursos humanos y financieros para realizar los procesos de identificación, internalización, difusión y explotación de los diferentes cúmulos de información y conocimiento derivados de la práctica médica.

La gestión de estos cúmulos mejora la habilidad de los médicos para tomar decisiones críticas y complejas en menor tiempo, mejorando los servicios de salud de la población localizada particularmente en áreas rurales pobres y de alta marginación, en las cuales el acceso a servicios médicos de alta especialidad es limitado. Además, la telemedicina permite la reconfiguración de espacios de capacitación, aprendizaje y difusión de conocimiento entre los médicos, especialmente a través de la teleeducación.

Finalmente, a pesar de la resistencia de algunos médicos al uso de nuevas tecnologías como la telemedicina, surgen cambios en las percepciones y en los patrones de comportamiento de los especialistas del ramo cuando ven los beneficios en los usuarios finales. De tal forma, cuando los modos de coordinación entre los diferentes especialistas tienden a ser menos jerárquicos y más horizontales, hay una probabilidad mayor de efectos positivos en los servicios de salud. Como se mostró anteriormente, estos modos de coordinación son influenciados por las estructuras de aprendizaje y transformación del conocimiento y no sólo por el hecho de adoptar la telemedicina. Esto puede darnos idea sobre los microfundamentos y su influencia en los cambios graduales de los modos de coordinación.

\section{CONCLusiones}

Las tecnologías sociales, como la telemedicina, adoptadas por las unidades médicas analizadas han sido absorbidas gradualmente. A pesar de la resistencia de una parte de la comunidad médica al uso de esa tecnología, las estructuras cognitivas desarrolladas previamente con el uso de las TIC permiten un incremento gradual de las capacidades de absorción en menor tiempo. Al mismo tiempo, esto ha implicado una reconfiguración de los procesos de aprendizaje, ya que la telemedicina ha implicado el desarrollo de la teleconsulta y la teleeducación como procesos de aprendizaje novedosos para el sector, particularmente para los médicos de menor especialidad. 
Esos cambios tienen implicaciones importantes en la transformación interna de las percepciones, preferencias y comportamiento de los actores involucrados en el proyecto, y a partir de ellos se reconfiguran los modos de coordinación, los cuales tienden a ser menos jerárquicos y más horizontales, aunque delimitados por las capacidades de los médicos para socializar el conocimiento y tomar decisiones complejas a través de la telemedicina.

Sin duda los procesos de absorción de la telemedicina tienen efecto sobre las relaciones intraorganizacionales al permitir una difusión de información y conocimiento específicos y la toma de decisiones críticas y complejas en menor tiempo, afectando el desarrollo de habilidades y conocimiento de los médicos y los servicios de salud otorgados a través de la teleconsulta. También afectan los modos de coordinación en el corto plazo, ya que cambian las percepciones y preferencias de los actores respecto al conocimiento y las formas en las que es socializado y explotado en el largo plazo, es decir, muestran un cambio microinstitucional que se podría reflejar en una mejora de la calidad de los servicios de atención a la salud.

\section{REFERENCIAS BIBLIOGRÁFICAS}

Arthur, B. (1994), Increasing returns and path dependence in the economy, Ann Arbor, University of Michigan Press.

Arrow, K. (1962), "Ecomomic welfare and the allocation of resources to invention", en R. Nelson (ed.), The rate and direction of inventive activity: economic and social factors, Princeton, Princeton University Press.

Ayala, E. José (2001), Economía del sector público mexicano, México, Esfinge-Feunam.

Bashshur, R.; Reardo, T. G., y Shannon, G. W. (1997), Telemedicine, theory and practice, , Springfield, Illinois, Charles C. Thomas Pub.

Bell, M., y Pavitt, K. (1995), “The Development of Technological Capabilities”, en I. Haque (ed.), Trade, Technology and International Competitiveness, Washington, The World Bank, pp. 69-101.

Bergh, J. C. van den, y Stagl, J. M. (2003), "Coevolution of economic behavior and institutions: toward a theory of institutional change", Journal of Evolutionary Economics, 13(3), pp. 289-317.

Bosch, F. van den; Volberda, H., y Boer, M. de (1999), "Coevolution of Firm Absorptive Capacity and Knowledge Environment: Organizational Forms and Combinative Capabilities", Organization Science, 10(5), pp. 551-568. 
Campbell, J. L. (2004), Institutional change and globalization, Princeton, Princeton University Press.

- (2007), "Challenges for institutional theory: A response to Crouch, Streeck, and Whitley”, Socio-Economic Review. Vol. 5, 556-567.

- (2009), "Surgimiento y transformación del análisis institucional", en Eduardo Ibarra C. (coord.), Estudios institucionales. Caracterización, perspectivas y problemas. La crisis de las instituciones modernas, México, UAM-GEDISA, pp. 3-34.

Cohen, W. M., y Levinthal, D. A. (1990), “Absorptive Capacity: A New Perspective on Learning and Innovation", Administrative Science and Quarterly, 35(1), 128152.

Commons, R. (2003), “Economía institucional”, Revista de Economía Institucional, vol. 5, núm 8, pp. 191-201.

CONAPO (2012), Dinámica demográfica de México 2000-2010, México, ConaPo.

Davenport, T. H., y Prusak, L. (1998), Working Knowledge: How Organizations Manage What They Know, Cambridge, Ma., Harvard Business School Press.

David, P. (1985), "Clio and the economics of QWERTY", American Economic Review, vol. 75, núm. 2, pp. 332-337.

Douglas, M. (1986), How institutions think, Syracuse, Syracuse University Press.

Fong, B.; Fong, A. C. M., y Li, C. K. (2011), Telemedicine technologies. Information technologies in medicine and telehealth, New Delhi, Wiley Publication.

Hage, J. (2006), "Institutional Change and Societal Change: The Impact of Knowledge Transformation", en J. Hage y M. Meeus (eds.), Innovation, Science and Institutional Change, USA, Oxford University Press, pp. 464-482.

Hollingsworth, J. R. (2006), “A Path-Dependent Perspective on Institutional and Organizational Factors Shaping Major Scientific discoveries", en J. Hage y M. Meeus (eds.), Innovation, Science and Institutional Change, UsA, Oxford University Press, pp. 423-464.

Hodgson, G. (2007), Economía institucional y evolutiva, México, UAM.

Jones, O., y Craven, M. (2001), Absorptive Capacity and New Organizational Capabilities: A TCS Case Study, working paper, The Business School of the Manchester Metropolitan University, No. wP01/02.

Julve, G.; Prats, L., y Comas, J. (2009), "Innovación como cambio institucional: una aproximación desde las teorías de la complejidad", Investigaciones Europeas de Dirección y Economía de la Empresa, 15(2), pp. 93-104.

Lane, P.J.; Koka, B., y Pathak, S. (2002), “A Thematic Analysis and Critical Assessment of Absorptive Capacity Research", Academy of Management Proceedings, vol. 2002, núm 1, pp. 1-7. 
Levi-Strauss (1966), The savage mind, Chicago, Chicago University Press.

Lund Vinding, A. (2004), "Interaction Between Firms and Knowledge Institutions", en Jesper L. Christensen y Bengt-Ake Lundvall (eds.), Product Innovation, Interactive Learning and Economic Performance, Research on Technological Innovation, Management and Policy, Volume 8, Bingley, Emerald Group Publishing Limited, pp. 257-283.

Lundvall, B. (1992), National System of Innovation, Towards a Theory of Innovation and Interactive Learning, London, Pinter Publisher.

Mill, J. S., (1998), On liberti and other essays, New York, Oxford University Press.

Molina, R. E., y Carvajal, C. (2009), "Sistemas de regulación en el mercado de medicamentos", en G. Correa L. (coord.), Sistemas de salud pública y bienestar social. México, UAM, pp. 13-44.

Molina, R. E., y Sánchez, E. I. (2009), "Financiamiento y equidad en los servicios de salud en México: El gasto de los hogares”, en G. Correa L. (coord.), Sistemas de salud pública y bienestar social. México, UAM, pp. 109-134.

Mosqueira, E., y Correa, G. (2009), "El sistema de salud en México y la epidemia del virus A/H1N1", en G. Correa L. (coord.), Sistemas de salud pública y bienestar social. México, UAM, pp. 75-108.

Nelson, R. (2002), "Bringing Institutions into Evolutionary Growth Theory”, Journal of Evolutionary Economics, 12(1), pp. 17-28.

Nelson, R., y Sampat, R. N. (2001), "Making Sense of Institutions as a Factor Shaping Economic Performance", Journal of Economic Behavior and Organization, 44(1), pp. 31-54.

Nelson, R., y Winter, S. (2002), "Evolutionary Theorizing in Economics", Journal of Economic Perspectives, American Economic Association, 16(2), pp. 23-46.

Nonaka, I., y Takeuchi, H. (1995), The Knowledge-Creating Company, usA, Oxford University Press.

North, D. (1990), Institutions, Institutional Change and Economic Performance, New York, Cambridge University Press.

Nugroho, Y. (2011), "Opening the black box: The adoption of innovation in the evolutionary sector - The case of Indonesia civil society organisations", Research Policy, Vol. 40, núm 5, pp. 761-777.

OECD (2011), “OECD.StatExtracts”, en Health Data 2011. Consultado en: http://stats.osd. org/index.aspx?DataSetCode=HEALTH_STAT.

oms (2000), Informe sobre la salud en el mundo, 2000, Suiza, Organización Mundial de la Salud 
— (2011), Estadísticas Sanitarias Mundiales, Francia, Organización Mundial de la Salud.

Polanyi, M. (2009), The Tacit Dimension, London, University of Chicago Press.

Paul, D. L. (2006), "Collaborative Activities in virtual Settings: A Knowledge Management Perspective of Telemedicine", Journal of Management Information System, 22(4), pp. 143-176.

Rogers, E. M. (2003), Diffusion of Innovation, 5th ed., s.1., New York Free Press.

Rooney, C. D. (1999), "Standing at the Millennium's Dawn: Telemedicine Leads Our Health Care System into the Twenty-First Century", Compensation \& Benefits Management, Autumn. 42-48.

Rosenberg, N. (1982), Inside the Black Box: Technology and Economics, Cambridge, Cambridge University Press.

Sampedro, J. L. (2011), Conocimiento y empresa: La industria del software en México, México, Plaza y Valdés/UAM-C.

Schotter, A. (1981), The economic theory of social institutions, New York, Cambridge University Press.

Senker, J., y Faulkner, W. (1996), “Networks, Tacit Knowledge and Innovation”, en R. Coombs, Al Richards, P. Saviotti y V. Walsh (eds.), Technological Collaboration. The Dynamics of Cooperation in Industrial Innovation, UK, Edward Elgar, pp. 76-97.

Smith, A. (1983), Investigación de la naturaleza y causas de la riqueza de las naciones, Barcelona, Orbis.

Veblen, T (2007), The theory of the leisure class, New York, Oxford University Press. 